\title{
The Matrix Transformations on Double Sequence Space of $\chi_{\pi}^{2}$
}

\author{
Nagarajan Subramanian And U.K. Misra
}

\begin{abstract}
Let $\chi^{2}$ denote the space of all prime sense double gai sequences and $\Lambda^{2}$ the space of all prime sense double analytic sequences. First we show that the set $E=\left\{s^{(m n)}: m, n=1,2,3, \cdots\right\}$ is a determining set for $\chi_{\pi}^{2}$. The set of all finite matrices transforming $\chi_{\pi}^{2}$ into FK-space $Y$ denoted by $\left(\chi_{\pi}^{2}: Y\right)$. We characterize the classes $\left(\chi_{\pi}^{2}: Y\right)$ when $Y=c_{0}^{2}, c^{2}, \chi^{2}, \ell^{2}, \Lambda^{2}$.

\begin{tabular}{|c|c|c|c|c|c|}
\hline$\nearrow$ & $c_{0}^{2}$ & $c^{2}$ & $\chi_{\pi}^{2}$ & $\ell^{2}$ & $\Lambda^{2}$ \\
\hline$\chi_{\pi}^{2}$ & \multicolumn{5}{|c|}{ Necessary and sufficient condition on the matrix are obtained } \\
\hline
\end{tabular}

But the approach to obtain these result in the present paper is by determining set for $\chi_{\pi}^{2}$. First, we investigate a determining set for $\chi_{\pi}^{2}$ and then we characterize the classes of matrix transformations involving $\chi_{\pi}^{2}$ and other known sequence spaces.
\end{abstract}

\section{INTRODUCTION}

Throughout $w, \chi$ and $\Lambda$ denote the classes of all, gai and analytic scalar valued single sequences respectively. We write $w^{2}$ for the set of all complex sequences $\left(x_{m n}\right)$, where $m, n \in \mathbb{N}$ the set of positive integers. Then $w^{2}$ is a linear space under the coordinate wise addition and scalar multiplication.

Some initial works on double sequence spaces is found in Bromwich [2]. Later on it was investigated by Hardy [3], Moricz [4], Moricz and Rhoades [5], Basarir and Solankan [1], Tripathy [6], Colak and Turkmenoglu [7], Turkmenoglu [8], and many others. We need the following inequality in the sequel of the paper. For $a, b, \geq 0$ and $0<p<1$, we have

$$
(a+b)^{p} \leq a^{p}+b^{p}
$$

The double series $\sum_{m, n=1}^{\infty} x_{m n}$ is called convergent if and only if the double sequence. $\left(s_{m n}\right)$ is called convergent, where $s_{m n}=\sum_{i, j=1}^{m, n} x_{i j}(m, n=$ $1,2,3, \ldots$ ) (see [9]). A sequence $x=\left(x_{m n}\right)$ is said to be double analytic if $\sup _{m n}\left|x_{m n}\right|^{1 / m+n}<\infty$. The vector space of all double analytic sequences

2000 Mathematics Subject Classification. Primary: 40A05, 40C05, 40 D05.

Key words and phrases. Determining set, gai sequence, analytic sequence, double sequence. 
will be denoted by $\Lambda^{2}$. A sequence $x=\left(x_{m n}\right)$ is called double gai sequence if $\left((m+n) !\left|x_{m n}\right|\right)^{1 / m+n} \rightarrow 0$ as $m, n \rightarrow \infty$. The double gai sequences will be denoted by $\chi^{2}$. Let $\phi=\{$ all finite sequences $\}$. Consider a double sequence $x=\left(x_{i j}\right)$. The $(m, n)^{t h}$ section $x^{[m, n]}$ of the sequence is defined by $x^{[m, n]}=\sum_{i, j=0}^{m, n} x_{i j} \Im_{i j}$ for all $m, n \in \mathbb{N}$,

$$
\Im_{m n}=\left(\begin{array}{cccccc}
0, & 0, & \cdots & 0, & 0, & \cdots \\
0, & 0, & \cdots & 0, & 0, & \cdots \\
\vdots & \vdots & \ddots & \vdots & \vdots & \\
0, & 0, & \cdots & \pi_{m n}, & -\pi_{m n}, & \cdots \\
0, & 0, & \cdots & 0, & 0, & \cdots
\end{array}\right)
$$

with $\pi_{m n}$ in the $(m, n)^{t h}$ position, $-\pi_{m n}$ in the $(m+1, n+1)^{t h}$ position and zero other wise. An FK-space (or a metric space) $X$ is said to have AK property if $\left(\Im_{m n}\right)$ is a Schauder basis for $X$. Or equivalently $x^{[m, n]} \rightarrow x$. An FDK-space is a double sequence space endowed with a complete metrizable; locally convex topology under which the coordinate mappings $x=\left(x_{k}\right) \rightarrow$ $\left(x_{m n}\right)(m, n \in \mathbb{N})$ are also continuous. If $X$ is a sequence space, we give the following definitions:

(i) $X^{\prime}=$ the continuous dual of $X$;

(ii) $X^{\alpha}=\left\{a=\left(a_{m n}\right): \sum_{m, n=1}^{\infty}\left|a_{m n} x_{m n}\right|<\infty\right.$, for each $\left.x \in X\right\}$;

(iii) $X^{\beta}=\left\{a=\left(a_{m n}\right): \sum_{m, n=1}^{\infty} a_{m n} x_{m n}\right.$ is convergent, for each $\left.x \in X\right\}$;

(iv) $X^{\gamma}=\left\{a=\left(a_{m n}\right): m, n \geq 1\left|\sum_{m, n=1}^{M, N} a_{m n} x_{m n}\right|<\infty\right.$, for each $\left.x \in X\right\}$;

(v) let $X$ be an $F K$-space $\supset \phi$; then $X^{f}=\left\{f\left(\Im_{m n}\right): f \in X^{\prime}\right\}$;

(vi) $X^{\Lambda}=\left\{a=\left(a_{m n}\right): \sup _{m n}\left|a_{m n} x_{m n}\right|^{1 / m+n}<\infty\right.$, for each $\left.x \in X\right\}$ $X^{\alpha} X^{\beta}, X^{\gamma}$ are called $\alpha$-(or Köthe-Toeplitz) dual of $X ; \beta$-(or generalizedKöthe-Toeplitz) dual of $X ; \gamma$-dual of $X, \Lambda$-dual of $X$ respectively.

\section{Definitions and Preliminaries}

$\chi_{\pi}^{2}=\left\{x=\left(x_{m n}\right):\left(\frac{x_{m n}}{\pi_{m n}}\right) \in \chi^{2}\right\} ;$

$\Lambda_{\pi}^{2}=\left\{x=\left(x_{m n}\right):\left(\frac{x_{m n}}{\pi_{m n}}\right) \in \Lambda^{2}\right\}$.

The space $\Lambda_{\pi}^{2}$ is a metric space with the metric

$$
d(x, y)=\sup _{m n}\left\{\left|\frac{x_{m n}-y_{m n}}{\pi_{m n}}\right|^{1 / m+n}: m, n: 1,2,3, \ldots\right\}
$$

for all $x=\left\{x_{m n}\right\}$ and $y=\left\{y_{m n}\right\}$ in $\Lambda^{2}$.

The space $\chi_{\pi}^{2}$ is a metric space with the metric

$$
d(x, y)=\sup _{m n}\left\{\left((m+n) !\left|\frac{x_{m n}-y_{m n}}{\pi_{m n}}\right|\right)^{1 / m+n}: m, n: 1,2,3, \ldots\right\}
$$


for all $x=\left\{x_{m n}\right\}$ and $y=\left\{y_{m n}\right\}$ in $\chi^{2}$.

Let $X$ be an BK-space. Then $D=D(X)=\{x \in \phi:\|x\| \leq 1\}$ we do not assume that $X \supset \phi$ (i.e.) $D=\phi \bigcap$ ( unit closed sphere in $X$ ).

Let $X$ be an BK space. A subset $E$ of $\phi$ will be called a determining set for $X$ if $D(X)$ is the absolutely convex hull of $E$. In respect of a metric space $(X, d), D=\{x \in \phi: d(x, 0) \leq 1\}$.

Given a sequence $x=\left\{x_{m n}\right\}$ and an four dimensional infinite matrix $A=\left(a_{m n}^{j k}\right), m, n, j, k=1,2, \ldots$ then $A$ - transform of $x$ is the sequence $y=\left(y_{m n}\right)$ when $y_{m n}=\sum_{m=1}^{\infty} \sum_{n=1}^{\infty} a_{m n}^{j k} x_{m n}(j, k=1,2, \ldots)$. Whenever $\sum \sum a_{m n}^{j k} x_{m n}$ exists.

Let $X$ and $Y$ be FK-spaces. If $y \in Y$ whenever $x \in X$, then the class of all matrices $A$ is denoted by $(X: Y)$.

Lemma 2.1. Let $X$ be a BK-space and $E$ is determining set for $X$. Let $Y$ be an FK-space and $A$ is an four dimensional infinite matrix. Suppose that either $X$ has $A K$ or $A$ is row finite. Then $A \in(X: Y)$ if and only if (1) The columns of $A$ belong to $Y$ and (2) $A[E]$ is a bounded subset of $Y$.

\section{Main Results}

Theorem 3.1. Let $E$ be the set of all sequences in $\phi$ each of whose non-zero terms is

$$
\left(\begin{array}{cccccc}
0, & 0, & \cdots & 0, & 0, & \cdots \\
0, & 0, & \cdots & 0, & 0, & \cdots \\
\vdots & \vdots & \ddots & \vdots & \vdots & \\
0, & 0, & \cdots & \frac{\pi_{m n}}{(m+n) !}, & \frac{-\pi_{m n}}{(m+n) !}, & \cdots \\
0, & 0, & \cdots & 0, & 0, & \cdots
\end{array}\right)
$$

with $\frac{\pi_{m n}}{(m+n) !}$, in the $(m, n)^{t h}, \frac{-\pi_{m n}}{(m+n) !}$, in the $(m+1, n+1)^{t h}$ position and zero other wise. Then $E$ is determining set of $\chi_{\pi}^{2}$.

Proof. Step 1. Recall that $\chi_{\pi}^{2}$ is a metric space with the metric

$$
d(x, y)=\sup _{m n}\left\{\left((m+n) !\left|\frac{x_{m n}-y_{m n}}{\pi_{m n}}\right|\right)^{1 / m+n}: m, n: 1,2,3, \ldots\right\}
$$

Let $A$ be the absolutely convex hull of $E$. Let $x \in A$.

Then $x=\sum_{m=1}^{i} \sum_{n=1}^{j} t_{m n} \pi_{m n} s^{(m n)}$ with

$$
\sum_{m, n=1}^{i, j}\left|t_{m n}\right| \leq 1
$$

and $s^{(m n)} \in E$.

Then $d(x, 0) \leq\left|t_{11}\right| \pi_{11} d\left(s^{(11)}, 0\right)+\cdots+\left|t_{i j}\right| \pi_{i j} d\left(s^{(i j)}, 0\right)$. But $d\left(s^{(m n)}\right)=$ 1 for $m, n=1,2,3, \ldots,(i, j)$. Hence $d(x, 0) \leq \sum_{m, n=1}^{i, j}\left|t_{m n}\right| \leq 1$ by using 
(4). Also $x \in \phi$. Hence $x \in D$. Thus

$$
A \subset D
$$

Step 2: Let $x \in D$

$\Rightarrow x \in \phi \quad$ and $\quad d(x, 0) \leq 1$.

$$
x=\left(\begin{array}{ccccc}
2 ! x_{11}, & 3 ! x_{12}, & \cdots & (1+n) ! x_{1 n}, & \ldots \\
3 ! x_{21}, & 4 ! x_{22}, & \cdots & (2+n) ! x_{2 n}, & \cdots \\
\vdots & \vdots & \ddots & \vdots & \\
(m+1) ! x_{m 1}, & (m+2) ! x_{m 2}, & \cdots, & (m+n) ! x_{m n}, & \ldots \\
0, & 0, & \cdots & 0, & \ldots
\end{array}\right)
$$

and

(6)

$$
\sup \left(\begin{array}{ccccc}
\left(2 !\left|x_{11}\right|\right)^{1 / 2}, & \left(3 !\left|x_{12}\right|\right)^{1 / 3}, & \cdots & \left((1+n) !\left|x_{1 n}\right|\right)^{1 / 1+n}, & \cdots \\
\vdots & \vdots & \ddots & \vdots & \\
\left((m+1) !\left|x_{m 1}\right|\right)^{1 / m+1}, & \left((m+2) !\left|x_{m 2}\right|\right)^{1 / m+2} & \cdots & \left((m+n) !\left|x_{m n}\right|\right)^{1 / m+n}, & \cdots \\
0, & 0, & \cdots & 0, & \cdots
\end{array}\right)
$$

Case (i): Suppose that $2 !\left|x_{11}\right| \geq \cdots \geq(m+n) !\left|x_{m n}\right|$. Take

Let $\xi_{m n}=\operatorname{Sgn}\left((m+n) ! x_{m n}\right)=\frac{(m+n) !\left|x_{m n}\right|}{(m+n) ! x_{m n}}$ for $m, n=1,2, \ldots,(i, j)$.

for $k, \ell=1,2,3, \ldots,(i, j)$.

$$
S_{k \ell} \pi_{k \ell}=\left(\begin{array}{ccccc}
\xi_{11}, & \xi_{12}, & \cdots & \xi_{1 \ell}, & \cdots \\
\xi_{21}, & \xi_{22}, & \cdots & \xi_{2 \ell}, & \cdots \\
\vdots & \vdots & \ddots & \vdots & \\
\xi_{k 1}, & \xi_{k 2}, & \cdots & \xi_{k \ell}, & \cdots \\
0, & 0, & \cdots & 0, & \cdots
\end{array}\right)
$$

Then $\pi_{k \ell} s_{k \ell} \in E$ for $k, \ell=1,2,3, \ldots,(i, j)$.

Also

$$
\begin{gathered}
x=\left(\left|2 ! x_{11}-3 ! x_{12}\right|-\left|3 ! x_{21}-4 ! x_{22}\right|\right) \pi_{11} S_{11}+\cdots \\
+\left(\left|(m+n) ! x_{m n}-(m+n+1) ! x_{m n+1}\right|\right. \\
\left.-\left|(m+n+1) ! x_{m+1 n}-(m+n+2) ! x_{m+1 n+1}\right|\right) \pi_{m n} S_{m n} \\
=t_{11} \pi_{11} S_{11}+\cdots+t_{m n} \pi_{m n} S_{m n},
\end{gathered}
$$

so that

$$
\begin{gathered}
t_{11}+\cdots+t_{m n}=\left|2 ! x_{11}-3 ! x_{12}\right| \\
-\left|(m+n+1) ! x_{m+1 n}-(m+n+2) ! x_{m+1 n+1}\right|=\left|2 ! x_{11}-3 ! x_{12}\right|
\end{gathered}
$$

because

$$
\left|(m+n+1) ! x_{m+1 n}-(m+n+2) ! x_{m+1 n+1}\right|=0 \leq 1
$$

by using (6). 
Hence $x \in A$. Thus $D \subset A$.

Case (ii): Let $y$ be $x$ and let $2 !\left|y_{11}\right| \geq \cdots \geq(m+n) !\left|y_{m n}\right|$. Express $y$ as a member of $A$ as in Case (i). Since $E$ is invariant under permutation of the terms of its members, so is $A$. Hence $x \in A$. Thus $D \subset A$. Therefore in both cases

$$
D \subset A
$$

From (5) and (7) $A=D$. Consequently $E$ is a determining set for $\chi_{\pi}^{2}$. This completes the proof.

Proposition 3.1. $\chi_{\pi}^{2}$ has $A K$.

Proof. Let $x=\left(x_{m n}\right) \in \chi_{\pi}^{2}$ and take $x^{[m n]}=\sum_{i, j=1}^{m, n} x_{i j} \Im_{i j}$ for all $m, n \in \mathbb{N}$. Hence $d\left(x, x^{[r s]}\right)=\sup _{m n}\left\{\left((m+n) !\left|\frac{x_{m n}}{\pi_{m n}}\right|\right)^{1 / m+n}: m \geq r+1, n \geq s+1\right\}$ $\rightarrow 0$ as $m, n \rightarrow \infty$

Therefore, $x^{[r s]} \rightarrow x$ as $r, s \rightarrow \infty$ in $\chi_{\pi}^{2}$. Thus $\chi_{\pi}^{2}$ has AK. This completes proof.

Proposition 3.2. An infinite matrix $A=\left(a_{m n}^{j k}\right)$ is in the class

$$
\begin{aligned}
& A \in\left(\chi_{\pi}^{2}: c_{0}^{2}\right) \Leftrightarrow \lim _{n, k \rightarrow \infty}\left(\pi_{m n} a_{m n}^{j k}\right)=0 \\
& \Leftrightarrow \sup _{m n}\left|\pi_{m 1} a_{m 1}^{j 1}+\cdots+\pi_{m n} a_{m n}^{j k}\right|<\infty .
\end{aligned}
$$

Proof. In Lemma 3 take $X=\chi_{\pi}^{2}$ has AK property and take $Y=\left(c_{0}^{2}\right)$ be an FK-space. Further more $\chi_{\pi}^{2}$ is a determining set $E$ (as in given Proposition 4). Also $A[E]=A\left(s^{(m n)}\right)=\left\{\left(\pi_{m 1} a_{m 1}^{j 1}+\cdots+\pi_{m n} a_{m n}^{j k}\right)\right\}$. Again by Lemma $3, A \in\left(\chi_{\pi}^{2}: c_{0}^{2}\right)$ if and only if:

(i) The columns of $A$ belong to $c_{0}^{2}$, and

(ii) $A\left(s^{(m n)}\right)$ is a bounded subset $\chi_{\pi}^{2}$.

But the condition

(i) $\Leftrightarrow\left\{\pi_{m n} a_{m n}^{j k}: j, k=1,2, \cdots\right\}$ is exits for all $m, n$;

(ii) $\Leftrightarrow \sup _{m n}\left|\pi_{m 1} a_{m 1}^{j 1}+\cdots+\pi_{m n} a_{m n}^{j k}\right|<\infty$.

Hence we conclude that $A \in\left(\chi_{\pi}^{2}: c_{0}^{2}\right) \Leftrightarrow$ conditions (8) and (9) are satisfied.

\section{The following proofs are similar. Hence we omit the proof.}

Proposition 3.3. An infinite matrix $A=\left(a_{m n}^{j k}\right)$ is in the class

$$
A \in\left(\chi_{\pi}^{2}: c^{2}\right) \Leftrightarrow \lim _{n, k \rightarrow \infty}\left(\pi_{m n} a_{m n}^{j k}\right) \operatorname{exists}(m, j=1,2,3, \ldots)
$$




$$
\Leftrightarrow \sup _{m n}\left|\pi_{m 1} a_{m 1}^{j 1}+\cdots+\pi_{m n} a_{m n}^{j k}\right|<\infty .
$$

Proposition 3.4. An infinite matrix $A=\left(a_{m n}^{j k}\right)$ is in the class

$$
A \in\left(\chi_{\pi}^{2}: \chi_{\pi}^{2}\right) \Leftrightarrow \sup _{m n}\left(\frac{1}{\pi_{m n}(m+n) !}\left|a_{m 1}^{j 1}+\cdots+a_{m n}^{j k}\right|\right)^{1 / m+n}<\infty .
$$

$$
\begin{gathered}
\Leftrightarrow \lim _{n, k \rightarrow \infty}\left(\frac{1}{\pi_{m n}(m+n) !}\left|a_{m n}^{j k}\right|\right)^{1 / m+n}=0, \text { for } m, j=1,2,3, \ldots \\
\Leftrightarrow d\left(a_{m 1}^{j 1}, a_{m 2}^{j 2}, \cdots, a_{m n}^{j k}\right) \text { is bounded } \\
\quad \quad \text { for each metric } d \text { on } \chi_{\pi}^{2} \text { and for all } m, n .
\end{gathered}
$$

Proposition 3.5. An infinite matrix $A=\left(a_{m n}^{j k}\right)$ is in the class

$$
\begin{gathered}
A \in\left(\chi_{\pi}^{2}: \ell^{2}\right) \Leftrightarrow \sum_{m=1}^{\infty} \sum_{n=1}^{\infty}\left|a_{m n}^{j k}\right| \text { converges }(j, k=1,2,3, \ldots) \\
\Leftrightarrow \sup _{m n} \sum_{m=1}^{\infty} \sum_{n=1}^{\infty}\left|\pi_{m n} a_{m n}^{j k}\right|<\infty
\end{gathered}
$$

Proposition 3.6. An infinite matrix $A=\left(a_{m n}^{j k}\right)$ is in the class

$$
\begin{gathered}
A \in\left(\chi_{\pi}^{2}: \Lambda^{2}\right) \Leftrightarrow \sup _{m n}\left(\left|\pi_{m n} \sum_{\gamma=1}^{n} \sum_{\mu=1}^{k} a_{m \gamma}^{j \mu}\right|^{1 / m+n}\right)<\infty \\
\Leftrightarrow d\left(a_{m 1}^{j 1}, a_{m 2}^{j 2}, \cdots a_{m n}^{j k}\right) \quad \text { is bounded } \\
\quad \text { for each metric } d \text { on } \Lambda^{2} \text { and for all } m, n .
\end{gathered}
$$

\section{REFERENCES}

[1] M. Basarir and O. Solancan, On some double sequence spaces, J. Indian Acad. Math., 21(2) (1999), 193-200.

[2] Bromwich, An introduction to the theory of infinite series Macmillan and Co.Ltd., New York, 1965.

[3] G.H. Hardy, On the convergence of certain multiple series, Proc. Camb. Phil. Soc., 19 (1917), 86-95.

[4] F. Moricz, Extention of the spaces $c$ and $c_{0}$ from single to double sequences, Acta. Math. Hungerica, 57(1-2), (1991), 129-136.

[5] F. Moricz and B.E. Rhoades, Almost convergence of double sequences and strong regularity of summability matrices, Math. Proc. Camb. Phil. Soc., 104, (1988), 283-294.

[6] B.C. Tripathy, On statistically convergent double sequences, Tamkang J. Math., 34(3), (2003), 231-237. 
[7] R. Colak and A. Turkmenoglu, The double sequence spaces $\ell_{\infty}^{2}(p), c_{0}^{2}(p)$ and $c^{2}(p)$, (to appear).

[8] A. Turkmenoglu, Matrix transformation between some classes of double sequences, Jour. Inst. of math. and Comp. Sci. (Math. Seri. ), 12(1), (1999), 23-31.

[9] T. Apostol, Mathematical Analysis, Addison-wesley, London, 1978.

[10] Erwin Kreyszig, Introductory Functional Analysis with Applications, John Wiley and Sons, 1978.

Nagarajan Subramanian
Department of Mathematics
SASTRA University
TANJORE-613 402
India
E-mail address: nsmaths@yahoo.com

U.K. MisRa

Department of Mathematics

BERHAMPUR UNIVERSITY

BERHAMPUR-760 007

ORISSA

INDIA

E-mail address: umakanta_misra@yahoo.com 